\title{
Full-Body Motion-Based Game Interaction for Older Adults
}

\author{
Kathrin M. Gerling ${ }^{1}$, Ian J. Livingston², Lennart E. Nacke ${ }^{3}$, Regan L. Mandryk ${ }^{4}$ \\ ${ }^{1}$ Interaction Lab, University of Saskatchewan, Saskatoon, SK, CA, kathrin.gerling@usask.ca \\ ${ }^{2}$ Playtest Lab, Ubisoft Divertissements Inc., Montréal, QC, CA, ian.livingston@ubisoft.com \\ ${ }^{3}$ University of Ontario Institute of Technology, Oshawa, ON, CA, lennart.nacke@uoit.ca \\ ${ }^{4}$ Interaction Lab, University of Saskatchewan, Saskatoon, SK, CA, regan@cs.usask.ca
}

\begin{abstract}
Older adults in nursing homes often lead sedentary lifestyles, which reduces their life expectancy. Full-body motion-control games provide an opportunity for these adults to remain active and engaged; these games are not designed with age-related impairments in mind, which prevents the games from being leveraged to increase the activity levels of older adults. In this paper, we present two studies aimed at developing game design guidelines for full-body motion controls for older adults experiencing agerelated changes and impairments. Our studies also demonstrate how full-body motion-control games can accommodate a variety of user abilities, have a positive effect on mood and, by extension, the emotional well-being of older adults. Based on our studies, we present seven guidelines for the design of full-body interaction in games. The guidelines are designed to foster safe physical activity among older adults, thereby increasing their quality of life.
\end{abstract}

\section{Author Keywords}

Older adults; games; entertainment; design.

\section{ACM Classification Keywords}

K.4.2 [Computers and Society]: Social Issues - Assistive technologies for people with disabilities, Handicapped persons/special needs; K.8.0 [Personal Computing]: General - Games.

\section{INTRODUCTION}

Older adults in nursing homes often lead sedentary lifestyles despite physical and cognitive activity being crucial for well-being in old age. Decreased activity adversely affects an individual's life expectancy, frequently leading to sedentary death syndrome [26]. Care facilities face the challenge of encouraging their residents to participate in leisure activities. Despite various efforts, few activities remain accessible, and it is difficult to motivate older adults to remain cognitively and physically active as the impact of age-related changes grows (e.g., cognitive impairments or mobility disabilities). Research has shown

Permission to make digital or hard copies of all or part of this work for personal or classroom use is granted without fee provided that copies are not made or distributed for profit or commercial advantage and that copies bear this notice and the full citation on the first page. To copy otherwise, or republish, to post on servers or to redistribute to lists, requires prior specific permission and/or a fee.

CHI'12, May 5-10, 2012, Austin, Texas, USA

Copyright 2012 ACM 978-1-4503-1015-4/12/05...\$10.00. that playing games can have positive effects on the emotional and physical well-being of elderly persons, and can motivate them to maintain a basic level of activity [4, 17]. The latest generation of game input devices, such as Microsoft Kinect, provides an opportunity for motivating physical activity. However, commercially available games put older adults at risk of injury by failing to accommodate for their range of abilities. In addition, there are no guidelines for designing gesture-based games for this particular group, and applying traditional $\mathrm{HCI}$ guidelines is difficult because games require special consideration to balance the difficulty needed to bring challenge - a necessary component of games - and ease of use.

We conducted two studies exploring the suitability of fullbody motion control for older adults. In our first study, we evaluated how well elderly participants could perform a set of gestures that was developed in collaboration with a physical therapist. Results suggest limitations in traditional gesture movements, and based on our findings in study one, we designed a game for institutionalized elderly using gestures for full-body motion control. In a second study we investigated how participants responded to the gestures in our game. Results from study two showed the success of our gestures, and demonstrated the positive effect of playing our game on participant mood. Our work makes three primary contributions: 1) We created a set of gestures suitable for institutionalized older adults in a gaming context, considering their range of motion, agility and strength; 2) We presented a full-body motion-controlled game for older adults and demonstrated that such games can have a positive effect on older adults' moods; influencing their well-being and providing enjoyable leisure activities; and 3) We present design guidelines for full-body motion controls to accommodate the range of abilities among institutionalized older adults. Additionally, we discuss how our findings and guidelines generalize beyond the scope of camera-based full-body motion-control games.

As our population ages, and digital entertainment systems become more pervasive, we can expect interest in video games among older adults to increase. Our work can expose the needs of older adults, but can also prepare practitioners to design full-body motion-control games for a broader audience with a range of physical and cognitive abilities. 


\section{RELATED WORK}

Game design for elderly persons has focused on design guidelines and case studies. The design of exergames for the elderly has been explored, but gesture-based input for games has only explored the context of younger players.

\section{Game Design for the Elderly}

First considerations on game design for elderly players date back to the 1980s, where Weisman [29] explored the accessibility of Apple II games among institutionalized older adults, highlighting the importance of adaptable games. More than 25 years later, the issue was addressed by Gamberini et al. [11], who focused on the impact of cognitive decrements on the use of entertainment systems, particularly addressing the design of graphical user interfaces. Likewise, Ijsselsteijn et al. [15] analyzed agerelated changes and present a set of design recommendations including the creation of individually meaningful and visually adjustable games providing multimodal feedback. Considerations of the design of meaningful games [25] highlight the importance of perceived benefits for the elderly. Additionally, Flores et al. [10] address the creation of games for stroke rehabilitation, recommending visual adaptability, inclusion of therapyappropriate movements, and suitable cognitive challenges. Different case studies have explored game design for older audiences. To motivate elderly persons to participate in rehabilitation, for example, after a stroke [2], game-based approaches have successfully been used. Often, Nintendo's Balance Board is used as an input device, for example, in various games for balance training [4]. Also, different approaches toward cognitive training for the elderly [3], and social interaction [19] have been explored. It has also been shown that institutionalized older adults enjoy playing games, but interaction challenges have to be addressed [13].

On closer examination, the design recommendations from previous work are high level, only providing small insights into the actual interaction between the older adult players and the system. Prior work has not explored the suitability of hardware game interfaces and associated interaction paradigms for older adults. Likewise, most case studies focus on game mechanics rather than addressing interaction design, frequently focusing on a limited body area. This has led to a lack of information regarding the application of full-body interaction for older adults.

\section{Designing Physical Interaction in Video Games}

Physical interaction in video games has been examined from the perspective of providing an exertive sports-like experience, or of exploring gesture-based interaction.

\section{Physical Activity Motivating Games}

Exergames integrate physical activity to engage the player [20]. Much work has focused on creating sports-like experiences to help fight sedentary lifestyles among younger audiences [7]. High-level considerations regarding exergames for elderly by Aarhus et al. [1] underline the importance of addressing individual differences by allowing players to adjust game speed and complexity. Further research by Gerling et al. [12] addresses the issue of game accessibility for institutionalized older adults by suggesting the support of standing and seated players to account for age-related impairments, and to avoid sudden and extensive movements. However, suitable interaction paradigms are not described. In the field of intergenerational play, it has been suggested that a design for enactive interaction and intuitive interfaces based on motor actions is required [27]. Yet, suitable gestures for game input are not provided.

\section{Gesture-based Interaction in Games}

Natural and gesture-based interaction has a history in 3D user interface design, and virtual environments [5, 14]. Existing efforts in those fields largely focus on productive tasks. However, design principles that focus on user enjoyment rather than task efficiency cannot be applied directly to the design of games. There is little research on full-body motion control in video games available. Despite the large popularity of gesture-based games, few have addressed gesture-based game control on an analytical level. For example, Payne et al. [22] discuss issues in the design of spatial 3D hand gestures in games, recommending the inclusion of tutorials, and highlighting the implementation of natural gestures. Work in full-body interaction for games has focused on the compilation of gesture recommendations and the exploration of player instruction. Norton et al. [21] provide a set of gestures for action games based on observations. Furthermore, they describe design guidelines for full-body interaction, which focus on steering and locomotion. Charbonneau et al. [6] explore player instruction and feedback visualization in dancing games. They match specific in-game actions, such as walking, with similar real-world movements. Their results show that movement visualization affects player performance, and timing is an issue in movement-based games, which is backed up by additional work by Johnston [16]. Rice et al. [23] present an evaluation of gesture-based mini games with 36 older adults. They suggest the exploration of gesture-based gaming for institutionalized older adults with a focus on hands free input.

Given that existing literature has only begun to analyze the needs of older adults specifically in game design, further research on interaction design for institutionalized older adults is necessary. This is particularly important, as effects of age-related changes are likely to affect the suitability of full-body interaction. Additionally, there remains a lack of research on the high-level accessibility of gestures. Interaction paradigms and game mechanics are often granted a higher priority than the consideration of individual player abilities, which is appropriate when designing for younger audiences but may not suffice when designing for older adults. We believe that reversing this philosophy - prioritizing ability before mechanics - makes games accessible to a larger audience. 


\section{FULL-BODY GAME INTERACTION FOR THE ELDERLY}

To explore full-body interaction for the elderly, considerations regarding the impact of age-related changes are necessary to address the special needs of this audience.

\section{Age-Related Changes and Impairments}

Age-related processes are likely to have an impact on older adults' ability to engage in gesture-based interaction. Common age-related changes include decreases in sensory acuity and effects on memory and attention [8]. Advanced age leads to the reduction of muscle mass, which causes decrements in strength and stamina [18]. The result is a lack of movement control and higher reaction and overall movement times [8]. Decrements in balance and gait are common among older adults, and the risk of falls increases [18]. Age is also suspected to cause difficulties in motor learning [18], which needs to be accounted for when designing movement-based interaction. Furthermore, older adults are likely to be affected by age-related diseases (e.g., arthritis or orthopedic impairments [8]), which affect their physical abilities and their capacity to use standard approaches to human-computer interaction. Cardiovascular diseases, such as stroke or heart attack, affect physical and cognitive abilities of older adults, leading to impairments [24]. All of those factors have to be considered when designing full-body interfaces for institutionalized older adults. First, the general accessibility of such interfaces has to be evaluated. Second, it is important to minimize the risk of injury due to unsuitable movements.

\section{A Gesture Set for Older Adults}

Based on the aforementioned considerations regarding agerelated changes, we suggest a basic gesture set (Table 1) that can be used to engage older adults in full-body interaction. It was created in cooperation with a physical therapy expert to ensure that all gestures are suitable for institutionalized older adults and do not overstrain users. The set includes a total of eight simple movements, which were derived from physical therapy exercises and everyday movements that institutionalized older adults are likely able to perform without much assistance. Postures and movements were chosen based on their accessibility for persons with limited physical abilities. We assume that integrating gestures that are intuitive and can easily be explained (e.g., clapping one's hands) offers the opportunity of easing older adults without prior computer/gaming experience into the interaction process.

The gesture set is limited to four core movements to account for a lack of previous activity as well as age-related impairments among the target audience, and to limit the amount of sensorimotor learning necessary to interact. Also, most gestures can be carried out while being seated to account for decrements in physical abilities (e.g., changes in posture and gait) and for the large amount of mobility disabilities among institutionalized older adults. In the set, we differentiate between static and dynamic gestures. Static gestures require users to hold a pose - for instance raising one arm and holding it up for a few seconds. Dynamic gestures require users to repeatedly carry out movements e.g., waving one arm instead of simply raising it. Due to the larger degree of activity required for dynamic gestures, we expected them to be more challenging.

\begin{tabular}{ccc}
\hline & Static & Dynamic \\
\hline Gesture 1 & Hands together & Clap hands \\
\hline Gesture 2 & Raise one arm & Wave arm \\
\hline Gesture 3 & Arms to the side & Pretend to fly \\
\hline Gesture 4 & One-leg stand & Walk in place \\
\hline
\end{tabular}

Table 1. Overview of static and dynamic gesture sets.

\section{STUDY 1: INPUT GESTURES FOR OLDER ADULTS}

In the first study, we investigated the suitability of the gesture set for institutionalized older adults. Furthermore, we explored performance differences between static and dynamic gestures, and the accessibility of interaction for older adults using Kinect.

\section{Gesture Analysis Tool}

To evaluate the feasibility of gesture-based interaction for institutionalized older adults using Kinect, we created a tool that prompts the user to perform our gesture set (Table 1). Player instruction was provided through descriptions, e.g., 'Put your hands together' or 'Wave your arm', and the display of a stick figure that performed the gesture. Users were provided with a green checkmark upon successful gesture completion. While users interacted with the tool, performance metrics (gesture completion, tracking quality) were logged. Gestures were tracked using relative joint positions. The tool was implemented in $\mathrm{C \#}$ using the Microsoft Kinect SDK and Microsoft Game Studio 4.0.

\section{Participants and Procedure}

Fifteen institutionalized older adults ( 7 female) participated in the evaluation, 13 living in a nursing home, and two older adults taking part in an adult day program at the same home. The mean age was 73.72 years $(\mathrm{SD}=9.90$; Range 60 to 90). Thirteen participants were in a wheelchair, one was using a cane for support while walking, and one person was able to walk independently. Six participants had experienced a stroke, leaving one side of their body paralyzed. Eight participants had played video games before, seven on a Wii. None of the participants had used camera-based systems such as Kinect before. The study was conducted at a media room of the nursing home that was equipped with a large-screen TV and offered enough space for participants to engage with the game. Participants first filled out a consent form and were informed about goals of the study. Then, demographic information was collected including information on the participant's health to ensure that the study would not put them at risk of injury. The gesture analysis tool was then introduced and participants were asked to perform the gesture set twice while being observed. Finally, participants completed a questionnaire on 
the suitability of the gesture set. The research protocol was approved by the research ethics boards at the University of Saskatchewan and the Saskatoon Health Region.

\section{Results}

\section{Questionnaire Results}

Participants were asked to rate their interaction experience on a 5-point Likert scale. Results show an overall positive experience regarding the fun users had while performing the gestures, and relatively low values for difficulty and perceived exhaustion (Table 2). 67\% of the participants were not afraid of losing balance, and $87 \%$ were not afraid of falling. $93 \%$ of the participants thought the program provided sufficient instructions, and $87 \%$ found that it provided enough feedback on gesture performance. Hand gestures were highlighted as easiest to perform, especially if movements were natural and already known from daily life, as one participant pointed out that "Raising my arm [was easiest] because it is just natural, it is quite strong.". Gestures that were affected by impairments were perceived as most difficult, mainly leg-based input and gestures requiring strength in both arms. Participants liked that the gestures allowed them to be active; some highlighted that they had to practice certain movements with their hands and that computer-based training might support that. Three participants stated that they did not like anything about the gestures as they were too simplistic. One participant highlighted that she would have liked to engage with a more complex system: "The gestures were not much fun, there was not much competition.".

\section{Performance Metrics}

Metrics were logged by the evaluation tool to track individual participant performance. The results show an overall completion rate of $54.17 \%$. Tracking gestures was sometimes not possible due to participants' limitations in range of motion or impairments; $16.67 \%$ of gestures were not recognized although carried out correctly. We found high completion rates for static hand-based gestures while rates for dynamic gestures were lower (Figure 1).

\section{Observations}

Observations during the first study revealed interaction differences among the target audience. Some of the participants could easily perform one gesture (e.g., raising

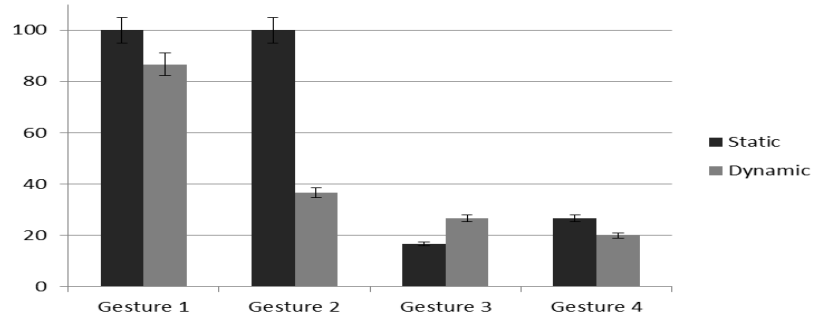

Figure 1. Completion results for gestures in percent (CI: 95\%)

\begin{tabular}{lcc}
\hline Item & Mean & SD \\
\hline Performing the gestures was fun. & 3.40 & 1.72 \\
\hline Performing the gestures was tiresome. & 1.87 & 1.41 \\
\hline Performing the gestures was easy. & 4.40 & 1.30 \\
\hline Performing the gestures was difficult. & 2.20 & 1.57 \\
\hline
\end{tabular}

Table 2. Mean results for items examining the perceived suitability of the gesture set $(5=$ completely agree).

one arm) but were unable to complete similar movements (e.g., moving one arm to the side). The wide range of impairments led to differences in gesture performances, which decreased the accuracy of our gesture recognition. We observed highly individual interaction styles due to varying physical abilities. For example, when players lifted their arm, there was a great variance in height. The ability to hold a posture or perform a gesture for a certain time also varied. Many participants required a long time to finish each gesture while others quickly completed each stage. For some of the participants, on-screen information was not sufficient and they turned to the investigators for assistance. Further observations revealed difficulties of the Kinect system dealing with participants in wheelchairs as the SDK only supports standing interaction. However, it was always possible to calibrate the system for users in wheelchairs.

\section{Findings}

Generally, institutionalized older adults represent an extremely heterogeneous target audience, which raises the necessity of adaptable interaction paradigms that can be individually adjusted. In our study, we found large differences between participant abilities resulting in differences in participant responses to our gesture set. Ability differences included limitations in range of motion (ROM), in strength affecting the ability of holding and repeating a gesture, and overall movement speed. Although the ability of using arms and legs was limited due to individual impairments that gesture-based interfaces need to account for, there are also main categories of institutionalized older adults ability that can be considered specifically, the ability of participants to use limbs on both sides of their body and the presence of wheelchairs. Completion rates suggest that dynamic gestures were more challenging for institutionalized older adults, perhaps because repeated movements are more demanding than holding a posture. Also, walking gestures were difficult for participants in wheelchairs; many of the participants were not able to move their feet independently. Generally, games need to work around the issue of wheelchair tracking by using space that is not affected by the chair, such as to the sides of the player, and removing bulky parts of the chair (e.g., lowering arm rests or removing lap trays) improves tracking results. Finally, being instructed by a computer screen was an unfamiliar experience for many participants.

\section{STUDY 2: GESTURE-BASED GAME INTERACTION}

In the second study, we explored the use of gestures in games addressing institutionalized older adults. Based on 
the results of the first study, we created a gesture-based game for institutionalized older adults. We then conducted a study using our game to refine gesture-based interaction for institutionalized older adults in games and to observe how the gesture-based game affected participant mood.

\section{A Gesture-Based Gaming System for Older Adults}

We created a gesture-based game that invites players to perform movements for growing a flower garden (Figure 2) because prior research suggested gardening themes for games for older adults [25]. The gesture system was designed to provide adaptable interaction paradigms that accommodated age-related changes, such as not being able to raise arms over a certain degree or allowing slower steady movements. The game was implemented in C\# using the Microsoft Kinect SDK and Microsoft Game Studio 4.0.

\section{Game Adjustment and Calibration}

Based on our findings from study one, basic information about the player's individual physical abilities needed to be provided upon game start to determine whether seated or standing interaction is possible, and if one-handed play is required. Then, players participated in a short calibration sequence during which they were asked to reach out to small flowers displayed on the screen. First, they were asked to collect as many flowers as possible to evaluate their ROM. Second, players had to reach to and hold certain flowers at specific screen positions to determine their strength and their ability of holding a certain pose. Player agility was calculated depending on completion time. Based on these values, players either engaged with the game via static or dynamic gestures. Threshold values for successful gesture completion and effect duration were set accordingly; if a player was assigned a low ROM value, the level of gesture precision was reduced. If players received low values for strength, game effects would remain active longer to avoid overexertion. Low agility values led to a larger time window for gesture completion.

\section{Gestures and Game Mechanics}

We developed four gesture-based game mechanics related to our gardening theme. These mechanics required players to perform different full-body gestures based on their individual abilities determined through calibration.

Growing plants. Players have to stand on one leg (static) or walk in place (dynamic) to grow plants. If players are seated, plants automatically grow.

Growing flowers. Players need to lift (static) or wave (dynamic) one arm. This activates rain that spreads from the player's hand onto the foliage.

Making flowers bloom. Flowers start blooming once players manage to get a certain amount of sunshine by either extending one or both arms to the side (static) or pretending to be flying by waving both arms (dynamic).

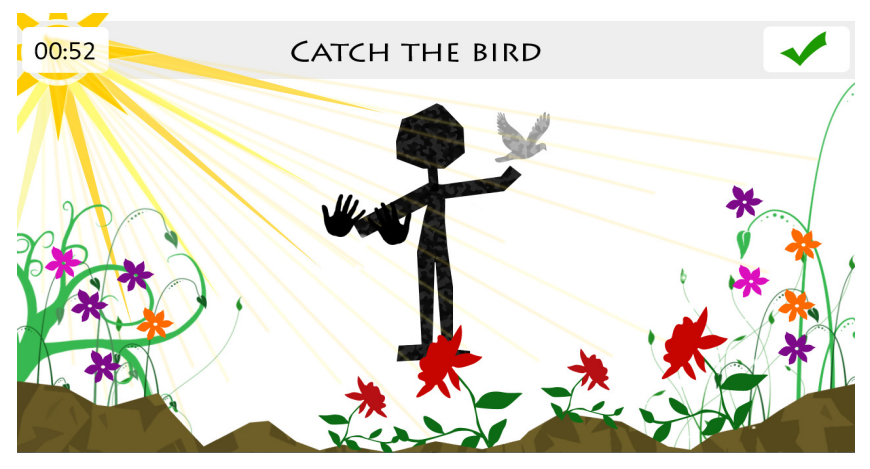

Figure 2. Screenshot of the tutorial phase of the game.

Catching the bird. Once all flowers have grown and are blooming, a bird appears. Players have to move their hand towards it to catch it, which provides an increase in pace.

\section{Instruction and Feedback: Guided and Free Play}

The first part of the game provided a period of guided tutorial play in which the game prompted players to perform certain gestures in a predefined order. Players were instructed in this tutorial by short phrases explaining the gesture and a stick figure demonstrating the movement. In the second part players could engage in free play, where they could perform any of the available gestures at any time without detailed instruction. When players successfully completed gestures, the gesture was revealed, and players were awarded points. The game used sound to underline the completion of player tasks. Once the garden was grown, a scoring screen was shown and the game ended.

\section{Participants and Procedure}

Twelve institutionalized older adults (5 female), with an average age of 76.7 ( $\mathrm{SD}=10.6$; Range 60 to 91), living in a nursing home participated in our study. Eleven participants were in wheelchairs; only one was able to walk without assistance. Similar to our first study, six participants reported having had a stroke, leaving one side of their body paralyzed. The study was conducted in the media-room of a nursing home. Participants first filled out a demographic questionnaire. Before participants started the game, they completed a pre-game PANAS questionnaire [28] to measure their positive and negative affect states. Participants first played the tutorial and then were allowed to engage in free play for five more minutes or until they successfully completed the game. After the session, they completed a post-game PANAS and gave feedback about the gestures and game mechanics.

\section{Results}

Based on the abilities of our study population, static gestures were assigned to all participants, hence we do not study differences between static and dynamic interaction.

Questionnaire Results

Differences in mood as assessed by the PANAS questionnaire before and after playing were analyzed using 


\begin{tabular}{lcc}
\hline Item & Mean & SD \\
\hline Performing the gestures was fun. & 3.08 & 1.62 \\
\hline Performing the gestures was tiresome. & 2.33 & 1.61 \\
\hline Performing the gestures was easy. & 3.83 & 1.03 \\
\hline Performing the gestures was difficult. & 2.75 & 1.29 \\
\hline
\end{tabular}

Table 3. Descriptive statistics for the perceived suitability of the gesture set used for the mini game ( 5 = completely agree).

a paired-samples t-test. There was a significant increase in positive affect after playing the game $(\mathrm{M}=3.88, \mathrm{SD}=0.79)$ compared to before the game $(\mathrm{M}=3.34, \mathrm{SD}=0.64), \mathrm{t}_{11}=-$ 2.92, $\mathrm{p}<0.01$. However, no changes in negative affect before $(M=1.72, S D=0.78)$ and after the game $(M=1.68$, $\mathrm{SD}=0.86), \mathrm{t}_{11}=0.28, \mathrm{p}=0.79$ were found. The questionnaires revealed that gestures were perceived as suitable, and the game was perceived as easy and fun (see Tables 3 and 4), yet we did not find any connections between player performance and these phenomena. Participants noted that the game had a positive effect on their alertness: "I' $m$ a little more attentive than I was before." Once used to the game, some participants highlighted that more feedback and more in-game options would improve their experience: "I wish more would have happened, like every time I moved." The participants generally liked the graphical style and theme of the game: "Oh, that [game] is cute."

\section{Performance Metrics}

The technical implementation of the Kinect game was successful as the performance logs showed no tracking failures. Completion results for hand and arm-based gestures were high (100\% for raising arm, $100 \%$ for moving $\operatorname{arm}(\mathrm{s})$ to the side(s), and $92 \%$ for catching the bird), whereas only one participant was able to perform foot-based gestures (Figure 5). Out of the twelve participants, eleven persons participated in free play.

\section{Observations}

Observations showed that most of the participants could interact with the game. Often, participants needed additional explanations. There were a few differences between guided play during the tutorial and the period of free play that followed it. Only some participants could correctly recall interaction paradigms after the tutorial and interact with game on their own, whereas others needed assistance from the experimenters to perform correct movements. Also, some participants showed physical fatigue after interacting with the game over longer periods of time. From a technical perspective, Kinect tracking worked well despite participants sitting in wheelchairs.

\section{Findings}

Based on the results, we conclude that institutionalized older adults generally enjoy engaging in full-body interaction games, and that the garden-themed game design presented in this paper appealed to the audience. When comparing the results of this study to study one, it becomes clear that providing an adaptable calibration routine largely

\begin{tabular}{lcc}
\hline Item & Mean & SD \\
\hline Playing the game was fun. & 3.79 & 0.99 \\
\hline Playing the game was tiresome. & 2.13 & 1.57 \\
\hline Playing the game was easy. & 3.71 & 1.48 \\
\hline Playing the game was difficult. & 1.83 & 1.11 \\
\hline
\end{tabular}

Table 4. Descriptive statistics for items examining the perceived suitability of the game $(5=$ completely agree $)$.

contributes to the accessibility of full-body motion-control for institutionalized older adults and enabled participants to engage in play regardless of individual impairments. However, results also show that most participants could not participate in free play on their own. This lends itself to the conclusion that free play is not suitable for elderly without prior gaming experience. For most of our players, recalling gestures was too challenging on their own. While proper game tutorials for institutionalized older adults remain to be explored in future work, one possible solution might be to introduce longer training periods for all gestures or designing game mechanics that suggest gesture affordances. For example, picking apples from a tree would trigger a known gesture and might be an easier game mechanic. Also, some of the participants seemed to be more alert and awake during the second part of the study, while others were less engaged. Therefore, an individual gesture adaptation is not only necessary to accommodate different players but also to account for the age-related within-player variances that we have observed in these studies.

\section{DESIGN GUIDELINES}

The results of the two studies presented in this paper show that accounting for the needs of institutionalized older adults in full-body game interaction requires considerations regarding their physical and cognitive abilities on various levels. We propose seven guidelines for the design of fullbody gestures for institutionalized older adults (Table 5) to support designers when creating entertainment software for nursing home environments. Each guideline addresses a specific problem in full-body game interaction for institutionalized older adults that we found during our studies or that was derived based on findings from the studies. Along with every guideline, we provide additional information on how it relates to the individual needs of institutionalized older adults, and we suggest possibilities of addressing the biggest design challenges.

In contrast to other work in the field of guidelines for activity motivating games for institutionalized older adults by Gerling et al. [12], which addresses high-level issues, our guidelines focus on the interaction process and provide detailed information regarding the most common interaction problems that we discovered during our studies. There are some similarities between our work and the guidelines for games for motor rehabilitation by Flores et al. [10], who recommend games that adapt to the motor skill level of players and the implementation of a therapyappropriate range of motion. However, they do not 


\section{Guideline 1: Age-Inclusive Design}

Create inclusive games by embracing age-related physical and cognitive impairments. Institutionalized older adults frequently experience ailments that prevent them from moving all of their limbs, which can severely influence the interaction process. Systems should account for this issue by including gestures that adapt to the player's individual impairments, e.g., by offering gestures which can be carried out using either one or both arms. Furthermore, the impact of cognitive changes has to be considered, e.g., by offering simpler game structures.

\section{Guideline 2: ROM-Adaptability}

Create interaction paradigms that adapt to individual differences in player range of motion. Institutionalized older adults often suffer from a reduced range of motion that limits their ability of engaging in full-body interaction. To account for this issue and to prevent injury, full-body interfaces for institutionalized older adults should be calibrated according to individual player abilities. Because limitations in player range of motion also lead to large differences in gesture execution, recognition in games for institutionalized older adults should feature a bigger tolerance in gesture execution instead of requiring a high level of precision for successful recognition.

\section{Guideline 3: Exertion Management}

Provide fatigue management and prevent overexertion by appropriate game pacing. Due to the prevalence of sedentary lifestyles, institutionalized older adults often have a reduced stamina level and are much more prone to movement based injury and overexertion. Games need to manage player fatigue through appropriate pacing, e.g., alternation of physically intense and less challenging game periods that allow players to relax and recover. Depending on the degree of player frailty, challenging periods should be shortened while relaxing game tasks can be extended. Reminders to take a break (or shutdown of the game) depending on the player's overall level of fitness should be integrated to avoid overexertion.

\section{Guideline 4: Dynamic Game Difficulty}

Offer difficulty adjustments between players and individually scale challenges. Games need to adjust to a large range in ability from one institutionalized older adult to another to allow for the appropriate level of activity and challenge to keep more active players engaged while avoiding overstraining others by dynamically adjusting the level of difficulty to player ability. Also, individual performance levels of institutionalized older adults change daily. Games need to account for this individual variability by dynamically adapting challenges for returning players instead of introducing a gradual increase of in-game challenges as the game progresses.

\section{Guideline 5: Easy Gesture Recall}

Provide natural mappings and clear instructions that support gesture recall to empower players. Many older adults have no prior experience playing games or being instructed through a computer screen, and are dependent on other persons for assistance when engaging in play. To address this problem, all instructions should be very clear and use common language. It is important to avoid the inclusion of information that is not crucial for play, e.g., additional GUI elements. Additionally, actions should be explainable using diagrams and simple on-screen demonstrations. To further support gesture learning and recall, in-game actions should map closely to real world activities. Games should not require players to recall a gesture; instead, players should be reminded of possible actions by affordances of in-game events, e.g., raising one's arm as an item appears at the top of the screen rather than raising one's arm to trigger another action.

\section{Guideline 6: Continuous Player Support}

Integrate continuous tutorials and player prompting to facilitate gesture learning and interaction. Much attention must be given to the training of institutionalized older adults. Extended tutorials are required to ensure that players are given time to learn the skills needed to play the game. This includes learning how to play the game in general and understanding how to use gestures to interact with it in particular. It is important that tutorials for gesture learning relate to previous knowledge to facilitate the acquisition of new skills and are repeated throughout gameplay to remind users of their options. Some older adults suffer from a reduced attention span that makes it difficult to follow an activity over a longer period. To grasp their attention, games should visually and audibly prompt the user if no interaction is detected. Also, it should not be assumed that players are capable of knowing when actions are required; they should constantly be prompted for correct input.

\section{Guideline 7: Simple Setup Routines}

Implement easy menus, startup and shutdown routines to encourage independent play. Technical knowledge cannot be assumed from either institutionalized older adults or nursing staff. Therefore, games must be easy to set up and run, but must also facilitate take down and cleanup. Due to the unfamiliarity that many institutionalized older adults have with this form of technology, the traditional traversal of a menu structure cannot be assumed as common knowledge.

Table 5. Design guidelines for full-body game interfaces for institutionalized older adults. Each guideline is supported by additional information on its background and how potential issues can be avoided. 
elaborate on how those goals can be achieved, and this information is provided by the guidelines presented in our paper. Further work by Gerling et al. [13] on the design of games for institutionalized older adults highlights the importance of meaningful metaphors on a general level, which we map onto full-body gaming.

Some of our guidelines relate to those provided by Norton et al. [21], who highlight the importance of player recall of in-game interaction options as well as accounting for player fatigue in game design. The guidelines presented in this paper expand on those aspects and elaborate on how they can be applied to interaction design for older adults.

\section{DISCUSSION}

The work presented in this paper looks specifically at the challenges of designing full-body motion-controlled games for institutionalized older adults. In the previous sections, we have demonstrated the need for specific considerations when designing motion-based games for elderly. In this section, we discuss the significance of our observations during the studies, the need for adaptable gestures and adaptable gesture systems, and the positive effect that gesture-based games may have on the emotional well-being of institutionalized older adults. Furthermore, we address the scope and generalizability of our guidelines in domains beyond institutionalized older adults, and the contribution of our work to the greater CHI community.

\section{Full-Body Motion-Controlled Gaming for Elderly}

With a growing number of senior citizens, games may contribute to their well-being in full-time care, as physical activity is crucial for well-being in old age. Yet, certain problems and limitations have to be considered.

\section{Implementing Games for the Elderly Using Kinect}

When we compare gesture-based interaction using the Kinect to that of other gesture-based interfaces, the Kinect has clear benefits over the competition. Compared to other devices such as the Nintendo Wii Remote or PlayStation Move controller, the lack of a handheld controller becomes a clear advantage. The use of the Kinect allows for more natural interactions, and frees designers from the worry of arthritic hands holding a controller. Players (who are not familiar with traditional handheld controllers in the case of institutionalized older adults) are able to interact with the game directly. The lack of controllers can (if designed correctly) simplify the interaction, and allow for more natural mappings, which ease the entry into play if institutionalized older adults lack gaming experience.

\section{Motion-Based Game Design for Older Adults}

Our research results show that the elderly can interact with games, enjoy playful activities and have the desire to stay more active, which can be supported by video games. However, common game design practices balance the challenge of a game around the abilities of younger players, and diverse physical and cognitive abilities of older adults make the use of commercially available games problematic. Therefore, gestures used in such games must be adaptable to facilitate all player types.

Based on the results of study one, we found that it is important to individually calibrate games according to a player's needs. Most Kinect games require players to stand, have use of two arms and legs, good vision, quick reactions, and knowledge of the interaction with video games. Therefore, current generations of full-body motioncontrolled games are inaccessible not only to institutionalized older adults but to many players, e.g., children experiencing mobility disabilities. One of the main issues is that commercially available games only calibrate games according to player position to optimize tracking results. We believe that this calibration process has to be augmented to provide an inclusive approach towards motion-based game design. By implementing a calibration routine that considers individual player abilities, we provide a means of including broad audiences in motion-based gaming. In study two, we demonstrated that this approach offers the opportunity of allowing older adults to engage with digital games, and we believe it may be useful for general audiences to adapt games to individual player skills.

\section{Gesture Design and Adaptability}

The results of our two studies revealed three main implications for gesture design for institutionalized older adults. 1) Static gestures were generally easier to perform and may therefore be more suitable because the movements are limited and the durations can be carefully controlled. Dynamic gestures, while still viable, put older adults at an increased risk of overexertion. Generally, both gesture types work if systems for older adults scale to their abilities. 2) Player range of motion, strength and general agility influence the interaction process. Systems need to focus on recognizing few, well-defined user actions instead of supporting a large number of gestures. Difficulties experienced by participants during our study illustrate the need for forgiving recognition mechanisms. 3) Being able to intuitively relate to everyday actions was highlighted as a positive aspect and increased the ability of participants to complete movements. It was also beneficial when describing interaction paradigms and instructing players. However, there is a risk of restricting motion controls too much. If controls are reduced too far then interaction becomes limited - insufficient motions remain to keep players from becoming bored. It falls to designers to carefully balance the needs of players and interactions. Fortunately, our guidelines can be used to help to strike the correct balance desired by designers.

\section{Effects of Motion-Controlled Games on Older Adults}

When we combine the potential cognitive and physical benefits of full-body motion controls, the large potential of games having a positive effect on institutionalized older adults is revealed. Perhaps the most valuable outcome from 
our studies was the positive effect playing video games had on the mood of the participants. In both studies, we found that participants enjoyed the experience of playing the game by indicating a more positive affective state. A prolonged positive affective state leads to an overall increase in positive mood [9]. Players who have a positive experience are more likely to continue to play, and return at a later point. In this context, it is important to highlight that we understand games as a means of supplementing the life of older adults. To preserve positive effects, we recommend applying games as a gateway to new experiences rather than a replacement of other leisure activities.

\section{Gesture and Guideline Generalizability}

The gestures and guidelines we have presented in this paper are specifically designed to aid designers to build games for older adults who experience a wide range of physical and cognitive age-related changes. However, the same gestures and guidelines can be applied, in a general way, to games that are designed with full-body motion control in mind. If our guidelines are used in the development of any game, then players with other impairments will be able to enjoy these games. For example, players in wheelchairs or who experience other physical limitations would still be able to participate in these games. Also, games for young children with little motor control would benefit from simple gesturebased controls.

While our studies have looked at motion control in a video game context, motion controls are likely to increase in popularity beyond the scope of video gaming. Gestures and guidelines can be applied in other areas where older adults are required to interact with information systems and generally contribute to facilitation of their interaction with information technology. For instance, the implementation of gestures in ambient-assisted living has the potential of supporting older adults when interacting with information systems in their home environment. Tasks such as the motion-based control of home electronics (e.g., controlling lights or television) could greatly facilitate everyday life. In this context, applying appropriate interaction paradigms, e.g., the gesture set provided in this paper, can support the independence of older adults and allow them to continue living in their home environment even when experiencing age-related changes. Furthermore, institutionalized older adults might benefit from gesture-based interaction in daily life if routine tasks such as opening or closing doors can be augmented by motion-controlled interaction.

\section{FUTURE WORK}

After our first investigation of motion-controlled full-body interaction for institutionalized older adults, many research questions remain to be addressed by future work. Research should explore on-screen instruction for institutionalized older adults. Our results suggest that it is likely that institutionalized older adults will generally respond more positively to guided experiences. Future studies will explore this question in greater detail to determine whether it is possible to design games for independent play. Furthermore, an evaluation of possible benefits of engaging with full-body motion-controlled games is necessary with a focus on the investigation of positive cognitive and physical effects. In terms of game design for older adults, we plan to implement the gesture set presented in this paper into an adhoc game for nursing homes designed to engage residents for a longer period of time. Finally, we aim to evaluate the effect of our guidelines in game design for older adults by investigating methods of application in game development.

\section{CONCLUSION}

Older adults in nursing homes frequently lead sedentary lifestyles and therefore experience a reduced quality of life leading to decreased life expectancy. Video games offer the opportunity of motivating institutionalized older adults to become more active. Over the past few years, there has been an increase in motion-controlled video games, which hold the promise of engaging users in physical activity. However, there has been little research regarding the design of motion-controlled games for diverse audiences, resulting in little understanding of how to ensure that interaction techniques are functional for older adults. Our work is a first step in this direction. Through our study of motioncontrol for institutionalized older adults, we have exposed the limitations of current design philosophies and highlighted design opportunities to facilitate the creation of accessible motion-controlled video games. If full-body motion-controls are to enjoy the same acceptance as other interaction techniques, we must ensure that games and other entertainment technologies remain an accessible and enjoyable activity for all audiences, including older adults.

\section{ACKNOWLEDGMENTS}

We thank Anne Nacke for assisting us in the development of senior-friendly interaction paradigms. Additionally, we thank Saskatoon Health Region and Sherbrooke Community Centre for supporting our research, and the participants for their contribution during the evaluation phase. We thank all of our CHI reviewers for their helpful comments and NSERC, SURFNET, and the GRAND NCE for funding.

\section{REFERENCES}

1. Aarhus, R., Groenvall, E., Larsen, S.B., and Wollsen, S. Turning training into play: Embodied gaming, seniors, physical training and motivation. Gerontechnology 10, 2 (2011), 110-120.

2. Baalam, M., Rennick Egglestone, S., Fitzpatrick, G., Rodden, T., Hughes, A.-M., Nind, T., Axelrod, L., Harris, E., Ricketts, I., Mawson, S., and Burridge, J. Motivating mobility: designing for lived motivation in stroke rehabilitation. In Proc.of CHI 2011, ACM(2011), 3073-3082. 
3. Baecker, R.M., Shim, N., Tonon, K., Pandeliev, V., Birnholtz, J., Stern, Y., Steinerman, J.R., and Moffatt, $\mathrm{K}$. 'Serious' online gaming environments to enhance brain fitness in senior citizens. Gerontechnology 9, 2 (2010), 190.

4. Bateni, H. Changes in balance in older adults based on use of physical therapy vs the Wii Fit gaming system: a preliminary study. Physiotherapy (2011).

5. Bowman, D., Kruijff, E., LaViola, J., and Poupyrev, I. $3 D$ User Interfaces: Theory and Practice. AddisonWesley, Redwood City, CA, USA, 2004.

6. Charbonneau, E., Miller, A., Wingrave, C., and LaViola Jr., J.J. Understanding Visual Interfaces for the Next Generation of Dance-Based Rhythm Video Games. In Proc. of Sandbox '09, ACM (2009), 119-126.

7. Consolvo, S., Everitt, K., Smith, I., and Landay, J.A. Design Requirements for Technologies that Encourage Physical Activity. In Proc. of CHI 2006, ACM (2006), 457-466.

8. Czaja, S.J. and Lee, C.C. Information Technology and Older Adults. In: Sears, A. \& Jacko, J.A. (Eds.): The Human-Computer Interaction Handbook. Lawrence Earlbaum Associates, New York and London (2006).

9. Ekman, P. Moods, Emotion and Traits. In: Ekman, P. \& Davidson, R.J. (Eds.): The Nature of Emotion. Oxford University Press: New York (1994).

10. Flores, E., Tobon, G., Cavallaro, E., Cavallaro, F.I., Perry, J.C., and Keller, T. Improving patient motivation in game development for motor deficit rehabilitation. In Proc. of ACE 2008, ACM (2008), 381-384.

11.Gamberini, L., Alcaniz, M., Barresi, G., Fabregat, M., Ibanez, F., and Prontu, L. Cognition, technology and games for the elderly: An introduction to ELDER GAMES Project. PsychNology 4, 3 (2006), 285-308.

12. Gerling, K.M., Schild, J., and Masuch, M. Exergame Design for Elderly Users: The Case Study of Silver Balance. In Proc. of ACE 2010, ACM (2010), 66-69.

13. Gerling, K.M., Schulte, F.P., and Masuch, M. Designing and Evaluating Digital Games for Frail Elderly Persons. In Proc. of ACE 2011, ACM(2011).

14.Hinckley, K., Pausch, R., Goble, J.C., and Kassell, N.F. A survey of design issues in spatial input. In UIST '94 Proc. of UIST 1994, ACM (1994), 213-222.

15.Ijsselsteijn, W., Nap, H.H., and de Kort, Y. Digital Game Design for Elderly Users. In Proc.of FuturePlay 2007, ACM (2007), 17-22 .

16. Johnston, H. and Whitehead, A. Pose Presentation for a dance-based massively multiplayer online exergame. Entertainment Computing, 2 (2011), 89-96.

17.Jung, Y., Li, K.J., Janissa, N.S., Gladys, W.L.C., Lee, K.M. Games for a Better Life: Effects of Playing Wii
Games on the Well-Being of Seniors in a Long-Term Care Facility. Proc. of IE '09, ACM (2009).

18. Ketcham, E. and Stelmach, G.E. Age-Related Declines in Motor Control. In: Birren, J.E. \& Schaie, K.W. (Eds.): Handbook of the Psychology of Aging. San Diego and London: Academic Press (2001).

19. Keyani, P., Hsieh, G., Mutlu, B., Easterday, M., and Forlizzi, J. DanceAlong: Supporting Positive Social Exchange and Exercise for the Elderly Through Dance. In Proc. of CHI EA 2005, ACM (2005), 1541-1544.

20. Mueller, F., Edge, D., Vetere, F., Gibbs, M.R., Agamanolis, S., Bongers, B., and Sheridan, J.G. Designing Sports: A Framework for Exertion Games. In Proc. of CHI 2011, ACM (2011), 2651-2660.

21.Norton, J., Wingrave, C.A., and LaViola Jr., J.J. Exploring Strategies and Guidelines for Developing Full Body Video Game Interfaces. In Proc. of FDG 2010, ACM (2010), 155-162.

22.Payne, J., Keir, P., Elgoyhen, J., McLundie, M., Naef, M., Horner, M., and Anderson, P. Gameplay issues in the design of spatial 3D gestures for video games. In Proc. of CHI 2006, ACM (2006), 1217-1222.

23.Rice, M., Wan, M., Foo, M.-H., Ng, J., Wai, Z., Kwok, J., Lee, S., and Teo, L. Evaluating Gesture-Based Games With Older Adults on a Large-Screen Display. In Proc. of Sandbox '11, Vancouver, Canada (2011), 17-24.

24. Rothenbacher, D. and Brenner, H. Cardiovascular Diseases Among Older Adults. In: Wahl, H.W., Brenner, H., Mollenkopf, H., Rothenbacher, D., \& Rott, R. (Eds.): The Many Faces of Health, Competence and Well-Being in Old Age. Dordrecht: Springer.

25.De Schutter, B. and Vanden Abeele, V. Meaningful Play in Elderly Life. In Proc. of ICA 2008, ICA (2008).

26. VanBeveren, P.J. and Avers, D. Exercise and Physical Activity for Older Adults. In: Guccione, A., Wong, R.A., \& Avers, D. (Eds.). Geriatric Physical Therapy. St. Louis: Elsevier (2012), 64-85.

27. Vanden Abeele, V. and De Schutter, B. Designing intergenerational play via enactive interaction, competition and acceleration. Personal and Ubiquitous Computing 2010, 14 (2010), 425-433.

28. Watson, D., Clark, L.A., and Tellegan, A. Development and validation of brief measures of positive and negative affect: The PANAS scales. Journal of Personality and Social Psychology 56, 6 (1988), 1063-1070.

29. Weisman, S. Computer Games for the Frail Elderly. Gerontologist 23, 4 (1983), 361-363.

30. Young, W., Ferguson, S., Brault, S., and Craig, C. Assessing and training standing balance in older adults: A novel approach using the 'Nintendo Wii' Balance Board. Gait \& Posture 33, 2 (2011), 303-305. 